Review

\title{
A Systematic Review of Persistent Symptoms and Residual Abnormal Functioning following Acute COVID-19: Ongoing Symptomatic Phase vs. Post-COVID-19 Syndrome
}

\author{
Glenn Jennings ${ }^{1,2, *}$, Ann Monaghan ${ }^{1,2}$ (D) Feng Xue ${ }^{1,2}$, David Mockler ${ }^{3}$ and Román Romero-Ortuño $1,2,4,5, *$ (i) \\ 1 Discipline of Medical Gerontology, School of Medicine, Trinity College Dublin, D02 R590 Dublin, Ireland; \\ ann.monaghan@tcd.ie (A.M.); fexue@tcd.ie (F.X.) \\ 2 The Irish Longitudinal Study on Ageing, Trinity College Dublin, D02 R590 Dublin, Ireland \\ Library Reader Services, Trinity College Dublin, D08 W9RT Dublin, Ireland; mocklerd@tcd.ie \\ 4 Mercer's Institute for Successful Ageing, St. James's Hospital, D08 NHY1 Dublin, Ireland \\ Global Brain Health Institute, Trinity College Dublin, D02 PN40 Dublin, Ireland \\ * Correspondence: gljennin@tcd.ie (G.J.); romeroor@tcd.ie (R.R.-O.)
}

\section{check for} updates

Citation: Jennings, G.; Monaghan, A.; Xue, F.; Mockler, D.;

Romero-Ortuño, R. A Systematic

Review of Persistent Symptoms and Residual Abnormal Functioning following Acute COVID-19: Ongoing Symptomatic Phase vs. Post-COVID-19 Syndrome. J. Clin. Med. 2021, 10, 5913. https:// doi.org/10.3390/jcm10245913

Academic Editor: Robert Flisiak

Received: 12 November 2021 Accepted: 14 December 2021 Published: 16 December 2021

Publisher's Note: MDPI stays neutral with regard to jurisdictional claims in published maps and institutional affiliations.

Copyright: (c) 2021 by the authors Licensee MDPI, Basel, Switzerland. This article is an open access article distributed under the terms and conditions of the Creative Commons Attribution (CC BY) license (https:// creativecommons.org/licenses/by/ $4.0 /)$.
Abstract: Objective: To compare the two phases of long COVID, namely ongoing symptomatic COVID-19 (OSC; signs and symptoms from 4 to 12 weeks from initial infection) and post-COVID-19 syndrome (PCS; signs and symptoms beyond 12 weeks) with respect to symptomatology, abnormal functioning, psychological burden, and quality of life. Design: Systematic review. Data Sources: Electronic search of EMBASE, MEDLINE, ProQuest Coronavirus Research Database, LitCOVID, and Google Scholar between January and April 2021, and manual search for relevant citations from review articles. Eligibility Criteria: Cross-sectional studies, cohort studies, randomised control trials, and case-control studies with participant data concerning long COVID symptomatology or abnormal functioning. Data Extraction: Studies were screened and assessed for risk of bias by two independent reviewers, with conflicts resolved with a third reviewer. The AXIS tool was utilised to appraise the quality of the evidence. Data were extracted and collated using a data extraction tool in Microsoft Excel. Results: Of the 1145 studies screened, 39 were included, all describing adult cohorts with long COVID and sample sizes ranging from 32 to 1733. Studies included data pertaining to symptomatology, pulmonary functioning, chest imaging, cognitive functioning, psychological disorder, and/or quality of life. Fatigue presented as the most prevalent symptom during both OSC and PCS at $43 \%$ and $44 \%$, respectively. Sleep disorder $(36 \% ; 33 \%)$, dyspnoea $(31 \% ; 40 \%)$, and cough $(26 \% ; 22 \%)$ followed in prevalence. Abnormal spirometry $\left(\mathrm{FEV}_{1}<80 \%\right.$ predicted) was observed in $15 \%$ and $11 \%$, and abnormal chest imaging was observed in $34 \%$ and $28 \%$, respectively. Cognitive impairments were also evident $(20 \% ; 15 \%)$, as well as anxiety $(28 \% ; 34 \%)$ and depression $(25 \% ; 32 \%)$. Decreased quality of life was reported by $40 \%$ in those with OSC and $57 \%$ with PCS. Conclusions: The prevalence of OSC and PCS were highly variable. Reported symptoms covered a wide range of body systems, with a general overlap in frequencies between the two phases. However, abnormalities in lung function and imaging seemed to be more common in OSC, whilst anxiety, depression, and poor quality of life seemed more frequent in PCS. In general, the quality of the evidence was moderate and further research is needed to understand longitudinal symptomatology trajectories in long COVID. Systematic Review Registration: Registered with PROSPERO with ID \#CRD42021247846.

Keywords: long COVID; COVID-19; ongoing symptomatic COVID-19; post-COVID-19 syndrome; fatigue; symptomatology

\section{Introduction}

On 11 March 2020, the World Health Organisation (WHO) Director-General declared the COVID-19 outbreak a global pandemic [1] and, as of December 2021, over 263 million positive cases and over 5 million deaths have been confirmed worldwide [2]. Caused 
by the novel severe acute respiratory syndrome coronavirus 2 (SARS-CoV-2), COVID-19 represents a highly heterogeneous disease affecting the respiratory tract and multiple other organ systems, with fever, fatigue, and cough presenting as the most prevalent symptoms [3]. Less commonly reported symptoms include hyposmia, dyspnoea, headache, sore throat, and dizziness. The severity of COVID-19 manifestations ranges from asymptomatic to severe, with acute presentations often requiring invasive ventilation or extended stays in intensive care for patients [4]. Overall, the acute COVID-19 phase typically endures for a period of up to 4 weeks from the onset of initial infection [5]. In a subset of patients, symptoms can persist beyond the 4-week acute COVID-19 period into a post-acute phase that has been termed as 'long COVID' [5]. Long COVID can be further distinguished as 'ongoing symptomatic COVID-19' (OSC) and 'post-COVID-19 syndrome' (PCS), terms that describe persistent signs and/or symptoms in the periods from 4 to 12 weeks and over 12 weeks post-infection onset, respectively [5].

Due to the recentness of the COVID-19 pandemic, and the initial focus of research being on the acute phase symptomatology and treatment, an accurate characterisation of long COVID symptomatology in its distinct phases has remained elusive [6]. Thus, in this systematic review, we aimed to characterise and compare the OSC and PCS phases of long COVID, with an emphasis on prevalence, symptomatology, pulmonary and cognitive functioning, mental health aspects, and quality of life.

\section{Methods and Materials}

\subsection{Protocol Registration}

The review protocol was registered with PROSPERO, the international prospective register of systematic reviews by the National Institute of Health Research (ID: CRD42021247846). The protocol can be accessed on the PROSPERO register [7].

\subsection{Search Strategy}

A search strategy was created by a medical librarian that included MeSH terminology related to "post-acute COVID-19", "long COVID", "prevalence", "symptomatology", "spirometry", "imaging", "cognitive", "psychological burden", and "quality of life". The full search strategy is shown in Supplementary Data S1. EMBASE, MEDLINE, ProQuest Coronavirus Research Database, LitCOVID, and Google Scholar were searched between January and April 2021, with the search being limited to articles published between March 2020 and April 2021. A manual search of review articles' reference lists was also conducted to identify relevant citations.

\subsection{Eligibility Criteria and Study Selection}

Studies with samples sizes of 30 or more participants aged at least 18 years old reporting data on long COVID symptomatology and/or general post-acute COVID-19 functioning were included in the review. In terms of study designs, cross-sectional studies, cohort studies, randomised control trials, and case-control studies were included, while meta-analyses, systematic reviews, narrative reviews, clinical trials, case studies and series, opinion pieces, and non-peer reviewed publications were excluded. Studies with a gender imbalance greater than $80: 20 \%$ in either direction were also excluded, as well as those reporting on specific cohorts (e.g., only patients with anosmia). Table 1 summarises the full eligibility criteria.

Citations generated from the search strategy were imported into a systematic review management tool, Covidence [covidence.org, accessed on 1 March 2021]. All duplicate imports were removed and initial screening was conducted by two independent reviewers, with conflicts resolved with a third reviewer. All texts were then further screened by a single reviewer and studies adhering to the inclusion criteria were included in the data extraction stage. Studies were selected in accordance with the PICOS framework (Participants, Interventions, Comparisons, Outcomes, and Study Design) based on the Preferred Reporting Items for Systematic Reviews and Meta-Analyses (PRISMA) guidelines [8]. 
Table 1. Eligibility Criteria for Studies and Participants.

\begin{tabular}{|c|c|c|}
\hline & Inclusion Criteria & Exclusion Criteria \\
\hline Study Topic & $\begin{array}{l}\text { Studies with participant data concerning } \\
\text { long COVID symptomatology and/or } \\
\text { general post-acute COVID-19 } \\
\text { functioning. }\end{array}$ & $\mathrm{N} / \mathrm{A}$ \\
\hline Study Design & $\begin{array}{l}\text { Cross-sectional studies, cohort studies, } \\
\text { randomised control trials, and } \\
\text { case-control studies. }\end{array}$ & $\begin{array}{l}\text { Meta-analyses, systematic reviews, narrative reviews, } \\
\text { clinical trials, case studies and series, opinion pieces, } \\
\text { and non-peer reviewed publications. }\end{array}$ \\
\hline Condition of Participants & $\begin{array}{l}\text { Participants who tested positive for } \\
\text { SARS-CoV-2 infection or were suspected } \\
\text { of SARS-CoV-2 infection. }\end{array}$ & $\begin{array}{l}\text { Participants recovered from acute COVID-19 (denoted } \\
\text { as } \geq 4 \text { weeks following symptom onset or hospital } \\
\text { admission; immediately following discharge from } \\
\text { hospital; or indicated as "recovered" by the respective } \\
\text { researchers). }\end{array}$ \\
\hline Sample Size & $\mathrm{N} / \mathrm{A}$ & Studies with less than 30 participants. \\
\hline Participant Age & $\mathrm{N} / \mathrm{A}$ & Participants younger than 18 years of age \\
\hline Participant Gender & $\mathrm{N} / \mathrm{A}$ & Studies with a gender imbalance greater than $80: 20 \%$. \\
\hline Other & $\mathrm{N} / \mathrm{A}$ & $\begin{array}{l}\text { Entire participant cohorts with a specific characteristic } \\
\text { (e.g., only patients with anosmia). }\end{array}$ \\
\hline
\end{tabular}

\subsection{Data Extraction}

The data from the included studies were extracted by a single reviewer using Microsoft Excel (Supplementary Data S2). Data included were as follows: (i) study details (i.e., first author, date of publication, country of authorship, the topic of the study, and study design); (ii) population details (i.e., sample size, mean/median age, gender proportion, eligibility criteria, acute COVID-19 hospitalisation status, and time post-COVID-19 onset); (iii) prevalence data of residual symptoms; and (iv) prevalence data of abnormal cognitive, pulmonary, and chest imaging findings, and poor mental health and quality of life data. Missing data were requested from the respective corresponding authors, if necessary.

The timepoints of assessment were adjusted for uniformity, with 'time' relating to the number of weeks following the initial onset of acute COVID-19. For studies that reported time following acute phase recovery or hospital discharge, a 4-week acute phase period was inserted in accordance with NICE guidelines [5]. The clinical data were then recorded as individual prevalences at single timepoints, with several prevalence points collected in longitudinal studies. Prevalences within 4-12 weeks and after 12 weeks were collated to produce a mean (+range) prevalence per symptom in the OSC and PCS phases, respectively. An overarching long COVID prevalence incorporating all the data per symptom was also calculated. Prevalence data were only recorded for either OSC and PCS in symptoms or abnormal traits identified at three or more distinct assessment timepoints. The entire data synthesis strategy was completed via Microsoft Excel.

\subsection{Quality Appraisal and Risk of Bias}

The AXIS Critical Appraisal Tool [9] was applied to each included study by two independent reviewers. For each study, a score out of 20 was generated and any disparities were resolved with a third reviewer.

\section{Results}

\subsection{Description of Included Studies}

A total of 1445 studies were retrieved from the online databases, with a further 37 identified through references of review articles. After 292 duplicates were removed, an initial screening of the remaining 1190 studies was conducted. 179 studies were included for further screening which produced a final list of 39 studies for data extraction [10-48]. A PRISMA flow diagram outlining the screening process is provided in Figure 1. 


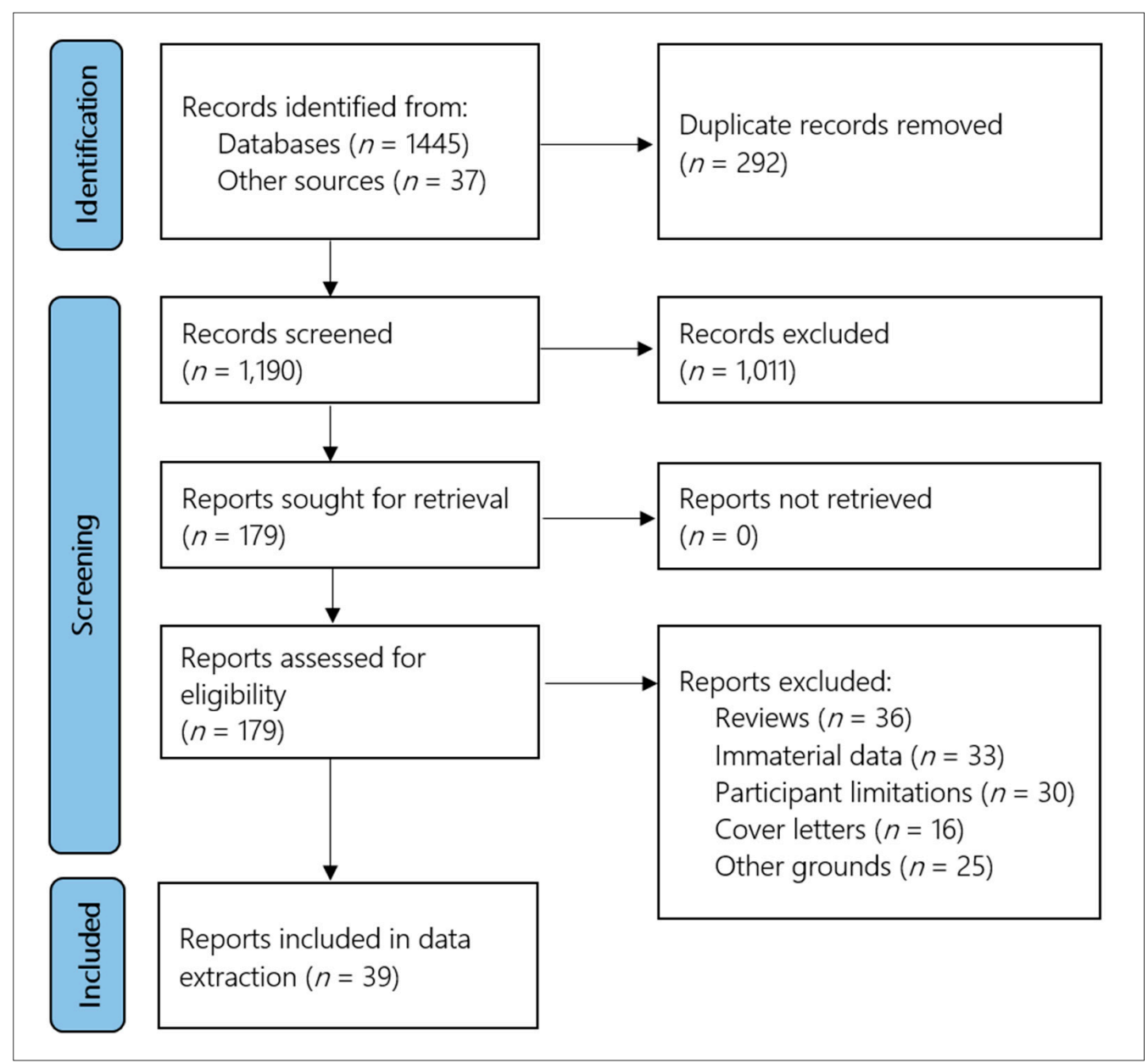

Figure 1. PRISMA flow diagram.

The main characteristics of the 39 included studies are presented in Table 2. Studies were conducted in 17 different countries. The sample sizes ranged from 32 to 1733, whilst participants' ages ranged from 32 to 74 years and proportions of female participants between $31 \%$ and $72 \%$. Participants' hospitalisation status varied between the studies, with $69 \%(n=27), 3 \%(n=1)$, and $28 \%(n=11)$ addressing inpatient, non-hospitalised, and mixed cohorts, respectively. Assessment time post-COVID-19 onset was between 4 and 31 weeks, with data available at 51 timepoints: 29 during OSC and 22 during PCS.

Table 2. Demographics of Included Studies.

\begin{tabular}{|c|c|c|c|c|c|c|c|}
\hline First Author & Date & Country & $\mathbf{N}$ & $\begin{array}{c}\text { Age } \\
\text { (Years) }\end{array}$ & $\begin{array}{c}\text { Gender } \\
\text { (\% Female) }\end{array}$ & $\begin{array}{c}\text { Participant } \\
\text { Hospital } \\
\text { Status }\end{array}$ & $\begin{array}{c}\text { Weeks from } \\
\text { COVID } \\
\text { Onset }\end{array}$ \\
\hline Arnold [10] & 21 April & $\begin{array}{c}\text { United } \\
\text { Kingdom }\end{array}$ & 110 & $M=60$ & $38 \%$ & Inpatient & 16 \\
\hline Bellan [11] & 21 January & Italy & 238 & $M=61$ & $40 \%$ & $\begin{array}{c}\text { Inpatient } \\
(+\mathrm{ICU})\end{array}$ & 21 \\
\hline Carfi [12] & 20 July & Italy & 143 & $\bar{X}=57$ & $37 \%$ & $\begin{array}{c}\text { Inpatient } \\
(+\mathrm{ICU})\end{array}$ & 9 \\
\hline Carvalho-Schneider [13] & 20 October & France & 150 & $\bar{X}=49$ & $56 \%$ & $\begin{array}{l}\text { Mixed } \\
\text { (-ICU) }\end{array}$ & 519 \\
\hline Cheng [14] & 21 January & $\begin{array}{l}\text { United } \\
\text { Kingdom }\end{array}$ & 113 & $M=73$ & $44 \%$ & $\begin{array}{c}\text { Inpatient } \\
(+\mathrm{ICU})\end{array}$ & 13 \\
\hline Chopra [15] & 20 November & America & 488 & $M=62$ & $48 \%$ & $\begin{array}{c}\text { Inpatient } \\
(+\mathrm{ICU})\end{array}$ & 13 \\
\hline
\end{tabular}


Table 2. Cont.

\begin{tabular}{|c|c|c|c|c|c|c|c|}
\hline First Author & Date & Country & $\mathbf{N}$ & $\begin{array}{c}\text { Age } \\
\text { (Years) }\end{array}$ & $\begin{array}{c}\text { Gender } \\
\text { (\% Female) }\end{array}$ & $\begin{array}{c}\text { Participant } \\
\text { Hospital } \\
\text { Status }\end{array}$ & $\begin{array}{c}\text { Weeks from } \\
\text { COVID } \\
\text { Onset }\end{array}$ \\
\hline Cortés-Telles [16] & 20 June & Mexico & 186 & $\bar{X}=47$ & $39 \%$ & Mixed & 9 \\
\hline Daher [17] & 20 October & Germany & 33 & $\bar{X}=64$ & $33 \%$ & Inpatient & 12 \\
\hline D'Cruz [18] & 21 January & $\begin{array}{l}\text { United } \\
\text { Kingdom }\end{array}$ & 119 & $\bar{X}=59$ & $38 \%$ & $\begin{array}{c}\text { Inpatient } \\
(+\mathrm{ICU})\end{array}$ & 13 \\
\hline De Lorenzo [19] & 20 October & Italy & 185 & $M=57$ & $34 \%$ & Mixed & 7 \\
\hline Froidure [20] & 21 April & Belgium & 134 & $M=60$ & $41 \%$ & $\begin{array}{c}\text { Inpatient } \\
(+\mathrm{ICU})\end{array}$ & 18 \\
\hline Garrigues [21] & 20 August & France & 120 & $\bar{X}=63$ & $37 \%$ & Mixed & 16 \\
\hline Halpin [22] & 21 February & $\begin{array}{l}\text { United } \\
\text { Kingdom }\end{array}$ & 100 & $R=20-84$ & $46 \%$ & Mixed & 11 \\
\hline Huang [23] & 21 January & China & 1733 & $M=57$ & $48 \%$ & $\begin{array}{c}\text { Inpatient } \\
(+\mathrm{ICU})\end{array}$ & 26 \\
\hline Huang [24] & 20 June & China & 57 & $\bar{X}=47$ & $54 \%$ & Inpatient & 8 \\
\hline Iqbal [25] & 21 February & Pakistan & 158 & $\bar{X}=32$ & $55 \%$ & Mixed & 7 \\
\hline Jacobs [26] & 20 December & America & 183 & $M=57$ & $38 \%$ & Inpatient & $4|6| 7 \mid 9$ \\
\hline Lerum [27] & 21 April & Norway & 103 & $M=59$ & $48 \%$ & Mixed & 12 \\
\hline Liang [28] & 20 October & China & 76 & $M=41$ & $72 \%$ & $\begin{array}{l}\text { Inpatient } \\
(+\mathrm{ICU})\end{array}$ & $5|13| 17$ \\
\hline Loerinc [29] & 21 March & America & 310 & $M=58$ & $51 \%$ & $\begin{array}{c}\text { Inpatient } \\
(+\mathrm{ICU})\end{array}$ & 4 \\
\hline Mandal [30] & 20 September & $\begin{array}{l}\text { United } \\
\text { Kingdom }\end{array}$ & 384 & $\bar{X}=60$ & $38 \%$ & $\begin{array}{c}\text { Inpatient } \\
(+\mathrm{ICU})\end{array}$ & 12 \\
\hline Miyazato [31] & 20 October & Japan & 63 & $\bar{X}=48$ & $33 \%$ & Inpatient & $9 \mid 17$ \\
\hline Mo [32] & 20 June & China & 110 & $\bar{X}=49$ & $50 \%$ & Inpatient & 4 \\
\hline Moreno-Perez [33] & 21 March & Spain & 277 & $M=62$ & $47 \%$ & Mixed & 11 \\
\hline Osikomaiya [34] & 21 March & Nigeria & 274 & $\bar{X}=42$ & $34 \%$ & Outpatient & 6 \\
\hline Prieto [35] & 21 March & Argentina & 85 & $\bar{X}=43$ & $45 \%$ & Mixed & 8 \\
\hline Raman [36] & 20 November & $\begin{array}{l}\text { United } \\
\text { Kingdom }\end{array}$ & 58 & $\bar{X}=55$ & $41 \%$ & $\begin{array}{c}\text { Inpatient } \\
(+\mathrm{ICU})\end{array}$ & 10 \\
\hline Rosales-Castillo [37] & 21 January & Spain & 118 & $\bar{X}=60$ & $44 \%$ & Inpatient & 11 \\
\hline Shah [38] & $21 \mathrm{March}$ & Canada & 60 & $M=67$ & $32 \%$ & Inpatient & 12 \\
\hline Simani [39] & 21 February & Iran & 120 & $\bar{X}=55$ & $33 \%$ & $\begin{array}{c}\text { Inpatient } \\
(+\mathrm{ICU})\end{array}$ & 30 \\
\hline Sykes [40] & 21 April & $\begin{array}{l}\text { United } \\
\text { Kingdom }\end{array}$ & 134 & $M=58$ & $34 \%$ & Mixed & $13|17| 20 \mid 25$ \\
\hline Taboada [41] & 20 Decenber & Spain & 91 & $\bar{X}=66$ & $35 \%$ & ICU & 30 \\
\hline Townsend [42] & 20 November & Ireland & 128 & $\bar{X}=50$ & $54 \%$ & Mixed & 14 \\
\hline Venturelli [43] & 21 January & Italy & 767 & $\bar{X}=63$ & $33 \%$ & $\begin{array}{c}\text { Inpatient } \\
(+\mathrm{ICU})\end{array}$ & 15 \\
\hline Walle-Hansen [44] & 21 March & Norway & 106 & $\bar{X}=74$ & $43 \%$ & $\begin{array}{c}\text { Inpatient } \\
(+\mathrm{ICU})\end{array}$ & 31 \\
\hline Wang [45] & 20 May & China & 131 & $M=49$ & $55 \%$ & Inpatient & $4|6| 8$ \\
\hline Wong [46] & 20 November & Canada & 78 & $\bar{X}=62$ & $36 \%$ & Inpatient & 13 \\
\hline Xiong [47] & 20 September & China & 538 & $M=52$ & $55 \%$ & Inpatient & 18 \\
\hline Yu [48] & 20 March & China & 32 & $\mathrm{M}=44$ & $31 \%$ & $\begin{array}{l}\text { Inpatient } \\
(+\mathrm{ICU})\end{array}$ & 5 \\
\hline
\end{tabular}

$\mathrm{N}=$ sample size. $\mathrm{M}=$ median. $\bar{X}=$ mean. $\mathrm{R}=$ range. $\mathrm{ICU}=$ intensive care unit.

\subsection{Quality Appraisal and Risk of Bias}

The average AXIS score for all included studies was $16.9( \pm 2.0)$ out of a possible 20, which may indicate a moderate risk of bias. The major sources of bias were the use of the convenience sampling methods, which was utilised by 38 of the 39 studies [10-24,26-49], and possible non-response bias in 12 studies [16,17,25-27,32,34-39,48]. The results of the AXIS critical appraisal for each included study are displayed in Table 3. 
Table 3. AXIS Critical Appraisal.

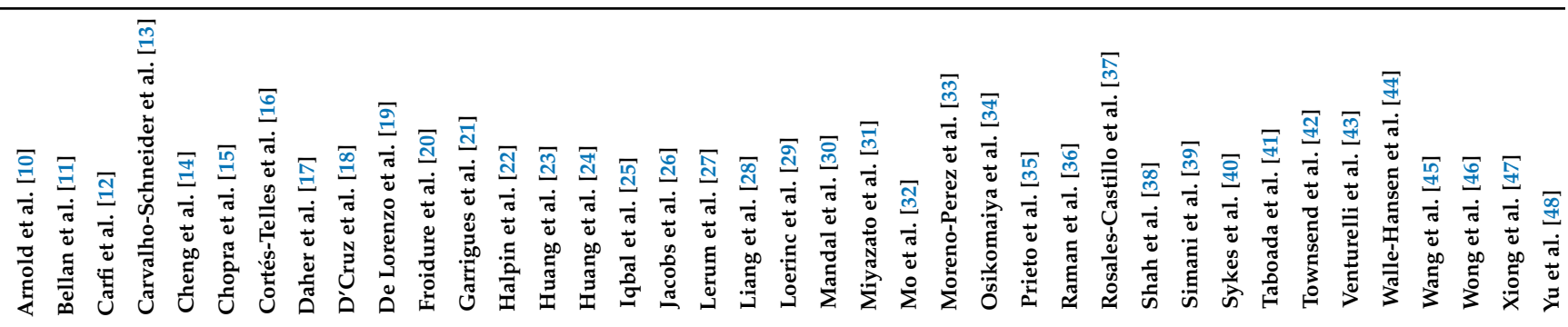

\begin{tabular}{|c|c|c|c|c|c|c|c|c|c|c|c|c|c|c|c|c|c|c|c|c|c|c|c|c|c|c|c|c|c|c|c|c|c|c|c|c|c|c|c|}
\hline Q1 & $\mathrm{N}$ & Y & $\mathrm{N}$ & Y & Y & Y & Y & Y & Y & Y & Y & $\mathrm{N}$ & $\mathrm{N}$ & Y & Y & Y & $\mathrm{N}$ & $\mathrm{N}$ & Y & Y & Y & $\mathrm{N}$ & Y & Y & Y & Y & Y & Y & $\mathrm{N}$ & Y & $\mathrm{N}$ & Y & Y & $\mathrm{N}$ & Y & Y & $Y$ & Y & Y \\
\hline Q2 & Y & Y & Y & Y & $Y$ & Y & Y & $Y$ & Y & Y & $Y$ & Y & Y & Y & $Y$ & Y & Y & Y & Y & Y & Y & Y & Y & Y & Y & Y & Y & Y & Y & $Y$ & Y & Y & Y & Y & Y & Y & $Y$ & Y & Y \\
\hline Q3 & $\mathrm{N}$ & $\mathrm{N}$ & $\mathrm{N}$ & $\mathrm{N}$ & $\mathrm{N}$ & $\mathrm{N}$ & $\mathrm{N}$ & $\mathrm{N}$ & $\mathrm{N}$ & $\mathrm{N}$ & $\mathrm{N}$ & $\mathrm{N}$ & $\mathrm{N}$ & $\mathrm{N}$ & $\mathrm{N}$ & $\mathrm{Y}$ & $\mathrm{N}$ & $\mathrm{N}$ & $\mathrm{N}$ & $\mathrm{N}$ & $\mathrm{N}$ & $\mathrm{N}$ & $\mathrm{N}$ & $\mathrm{N}$ & $\mathrm{N}$ & $\mathrm{N}$ & $\mathrm{N}$ & $\mathrm{N}$ & $\mathrm{N}$ & $\mathrm{N}$ & $\mathrm{N}$ & $\mathrm{N}$ & $\mathrm{N}$ & $\mathrm{N}$ & $\mathrm{N}$ & $\mathrm{N}$ & $\mathrm{N}$ & $\mathrm{N}$ & $\mathrm{N}$ \\
\hline Q4 & Y & Y & Y & Y & $Y$ & $\mathrm{~N}$ & Y & $Y$ & $\mathrm{Y}$ & Y & $Y$ & $\mathrm{~N}$ & Y & Y & $Y$ & Y & Y & Y & Y & Y & Y & Y & Y & Y & Y & Y & Y & $Y$ & Y & Y & Y & $\mathrm{Y}$ & Y & Y & Y & Y & Y & Y & Y \\
\hline Q5 & Y & Y & $Y$ & Y & $Y$ & $\mathrm{~N}$ & Y & $Y$ & Y & $Y$ & $Y$ & $\mathrm{~N}$ & Y & Y & $Y$ & Y & $Y$ & Y & Y & Y & Y & $Y$ & Y & Y & Y & Y & Y & Y & Y & Y & $Y$ & Y & Y & Y & Y & Y & $Y$ & $Y$ & Y \\
\hline Q6 & Y & Y & Y & Y & $Y$ & $\mathrm{~N}$ & Y & $\mathrm{N}$ & Y & Y & Y & $\mathrm{N}$ & Y & Y & $Y$ & Y & Y & Y & Y & Y & Y & Y & Y & Y & Y & Y & Y & Y & Y & Y & Y & Y & Y & Y & Y & Y & Y & Y & Y \\
\hline Q7 & $Y$ & $\mathrm{Y}$ & $\mathrm{Y}$ & $\mathrm{Y}$ & $Y$ & $\mathrm{~N}$ & $\mathrm{~N}$ & $Y$ & Y & $\mathrm{N}$ & $Y$ & Y & Y & Y & $Y$ & $\mathrm{~N}$ & $\mathrm{~N}$ & $\mathrm{~N}$ & Y & Y & Y & $Y$ & $\mathrm{~N}$ & $Y$ & $\mathrm{~N}$ & $\mathrm{~N}$ & $\mathrm{~N}$ & $\mathrm{~N}$ & $\mathrm{~N}$ & Y & Y & Y & Y & $Y$ & Y & $\mathrm{N}$ & Y & Y & $\mathrm{N}$ \\
\hline Q8 & $\mathrm{Y}$ & Y & Y & Y & $Y$ & Y & Y & $\mathrm{Y}$ & $\mathrm{Y}$ & Y & Y & $\mathrm{N}$ & Y & Y & $Y$ & Y & Y & Y & Y & $Y$ & Y & Y & Y & Y & Y & Y & $\mathrm{Y}$ & Y & Y & $\mathrm{Y}$ & Y & Y & Y & Y & Y & Y & Y & $\mathrm{Y}$ & Y \\
\hline Q9 & $Y$ & Y & Y & $\mathrm{Y}$ & $Y$ & Y & $Y$ & $Y$ & $\mathrm{Y}$ & Y & Y & $\mathrm{N}$ & Y & Y & Y & Y & $Y$ & Y & Y & Y & Y & $\mathrm{N}$ & Y & Y & Y & $\mathrm{Y}$ & $\mathrm{Y}$ & $Y$ & Y & Y & Y & Y & Y & Y & Y & Y & Y & Y & Y \\
\hline Q10 & $\mathrm{Y}$ & Y & Y & Y & $\mathrm{N}$ & Y & Y & $Y$ & Y & Y & Y & Y & Y & Y & $Y$ & Y & $Y$ & Y & Y & Y & $\mathrm{N}$ & $\mathrm{N}$ & Y & $\mathrm{Y}$ & Y & Y & Y & $Y$ & Y & Y & $\mathrm{N}$ & $\mathrm{N}$ & Y & Y & Y & $Y$ & $\mathrm{~N}$ & $Y$ & Y \\
\hline Q11 & $\mathrm{Y}$ & $\mathrm{Y}$ & Y & $\mathrm{Y}$ & Y & $\mathrm{N}$ & Y & $\mathrm{N}$ & $\mathrm{Y}$ & Y & Y & Y & Y & Y & $\mathrm{Y}$ & Y & $Y$ & Y & Y & Y & $\mathrm{N}$ & $\mathrm{N}$ & Y & $\mathrm{Y}$ & Y & Y & $\mathrm{Y}$ & $\mathrm{N}$ & Y & Y & Y & Y & Y & Y & Y & Y & $Y$ & $\mathrm{Y}$ & Y \\
\hline Q12 & $Y$ & Y & Y & Y & $Y$ & Y & $Y$ & $Y$ & Y & Y & $Y$ & Y & Y & Y & $Y$ & Y & $Y$ & Y & Y & Y & Y & $\mathrm{Y}$ & Y & $Y$ & Y & Y & $\mathrm{Y}$ & Y & Y & $Y$ & Y & Y & Y & Y & Y & Y & $Y$ & Y & Y \\
\hline Q13 & $\mathrm{N}$ & $\mathrm{N}$ & $\mathrm{N}$ & $\mathrm{N}$ & $\mathrm{N}$ & $\mathrm{N}$ & Y & $\mathrm{Y}$ & $\mathrm{N}$ & $\mathrm{N}$ & $\mathrm{N}$ & $\mathrm{N}$ & $\mathrm{N}$ & $\mathrm{N}$ & $\mathrm{N}$ & Y & Y & Y & $\mathrm{N}$ & $\mathrm{N}$ & $\mathrm{N}$ & $\mathrm{N}$ & $\mathrm{N}$ & $\mathrm{N}$ & Y & Y & $\mathrm{Y}$ & Y & Y & Y & $\mathrm{N}$ & $\mathrm{N}$ & $\mathrm{N}$ & $\mathrm{N}$ & $\mathrm{N}$ & $\mathrm{N}$ & $\mathrm{N}$ & $\mathrm{N}$ & Y \\
\hline Q14 & Y & $Y$ & Y & $\mathrm{Y}$ & $Y$ & Y & $\mathrm{N}$ & $\mathrm{N}$ & Y & $\mathrm{N}$ & $Y$ & Y & Y & Y & $Y$ & $\mathrm{~N}$ & $\mathrm{~N}$ & $\mathrm{~N}$ & Y & $\mathrm{Y}$ & Y & Y & $\mathrm{N}$ & Y & $\mathrm{N}$ & $\mathrm{N}$ & $\mathrm{N}$ & $\mathrm{N}$ & $\mathrm{N}$ & Y & $\mathrm{N}$ & Y & Y & Y & Y & Y & Y & Y & $\mathrm{N}$ \\
\hline Q15 & $Y$ & $Y$ & $Y$ & Y & $Y$ & $Y$ & $Y$ & $Y$ & $Y$ & $Y$ & $Y$ & $Y$ & $Y$ & $Y$ & $Y$ & Y & $\mathrm{Y}$ & $Y$ & Y & $Y$ & $Y$ & $Y$ & Y & $Y$ & Y & Y & $\mathrm{Y}$ & $\mathrm{N}$ & Y & $Y$ & Y & Y & $Y$ & $Y$ & Y & Y & $Y$ & Y & Y \\
\hline Q16 & $Y$ & Y & Y & Y & $Y$ & $Y$ & $Y$ & $Y$ & Y & Y & $Y$ & $Y$ & $Y$ & Y & $Y$ & Y & Y & Y & Y & $Y$ & Y & Y & Y & $Y$ & Y & Y & Y & Y & Y & Y & Y & Y & Y & Y & Y & Y & Y & $Y$ & Y \\
\hline Q17 & $Y$ & $Y$ & Y & Y & $Y$ & Y & Y & $Y$ & Y & $Y$ & $Y$ & $Y$ & Y & Y & $Y$ & Y & $Y$ & Y & Y & $Y$ & Y & Y & $Y$ & Y & Y & Y & Y & Y & Y & Y & Y & Y & Y & Y & Y & Y & Y & $Y$ & Y \\
\hline Q18 & $Y$ & Y & $\mathrm{Y}$ & $\mathrm{N}$ & $Y$ & Y & $Y$ & $\mathrm{~N}$ & Y & Y & Y & Y & Y & $Y$ & $Y$ & Y & $\mathrm{N}$ & $Y$ & Y & Y & Y & Y & Y & Y & Y & $\mathrm{Y}$ & Y & Y & Y & Y & Y & Y & Y & Y & Y & Y & Y & $\mathrm{N}$ & Y \\
\hline Q19 & $\mathrm{N}$ & Y & $\mathrm{N}$ & $\mathrm{N}$ & $\mathrm{N}$ & $\mathrm{N}$ & $\mathrm{N}$ & $\mathrm{N}$ & $\mathrm{N}$ & $\mathrm{N}$ & $\mathrm{N}$ & $\mathrm{N}$ & $\mathrm{N}$ & $\mathrm{N}$ & $\mathrm{N}$ & $\mathrm{N}$ & $\mathrm{N}$ & $\mathrm{N}$ & $\mathrm{N}$ & $\mathrm{N}$ & $\mathrm{N}$ & $\mathrm{N}$ & $\mathrm{N}$ & $\mathrm{N}$ & $\mathrm{N}$ & $\mathrm{N}$ & $\mathrm{N}$ & Y & $\mathrm{N}$ & $\mathrm{N}$ & $\mathrm{N}$ & $\mathrm{N}$ & $\mathrm{N}$ & $\mathrm{N}$ & $\mathrm{N}$ & $\mathrm{N}$ & $\mathrm{N}$ & $\mathrm{N}$ & $\mathrm{N}$ \\
\hline Q20 & Y & $\mathrm{Y}$ & Y & Y & $Y$ & $\mathrm{~N}$ & Y & $\mathrm{Y}$ & Y & Y & $\mathrm{Y}$ & $\mathrm{N}$ & Y & Y & Y & Y & Y & Y & Y & $\mathrm{Y}$ & $\mathrm{N}$ & Y & Y & Y & Y & Y & Y & $\mathrm{N}$ & Y & Y & $\mathrm{N}$ & Y & Y & Y & Y & Y & Y & Y & Y \\
\hline
\end{tabular}

$\mathrm{Q}=$ question. $\mathrm{Y}$ = yes. $\mathrm{N}=$ no. $\mathrm{Q} 1$. Were the aims/objectives of the study clear? $\mathrm{Q} 2$. Was the study design appropriate for the stated $\operatorname{aim}(\mathrm{s})$ ? Q3. Was the sample size justified? Q4. Was the target/reference population clearly defined? Q5. Was the sample frame taken from an appropriate population base that it closely represented the target/reference population under investigation? Q6. Was the selection process likely to select subjects/participants that were representative of the target/reference population under investigation? Q7. Were measures undertaken to address and categorise non-responders? Q8. Were the risk factor and outcome variables measured appropriate to the aims of the study? Q9. Were the risk factor and outcome variables measured correctly using instruments/measurements that had been trialled, piloted, or published previously? Q10. Is it clear what was used to determine statistical significance and/or precision estimates? Q11. Were the methods (including statistical methods) sufficiently described to enable them to be repeated? Q12. Were the basic data adequately described? Q13. Does the response rate raise concerns about non-response bias? Q14. If appropriate, was information about non-responders described? Q15. Were the results internally consistent? Q16. Were the results for the analyses described in the methods, presented? Q17. Were the authors' discussions and conclusions justified by the results? Q18. Were the limitations of the study discussed? Q19. Were there any funding sources or conflicts of interest that may affect the authors' interpretation of the results? Q20. Was ethical approval or consent of participants attained?

\subsection{Ongoing Symptomatic COVID-19 and Post-COVID-19 Syndrome}

Based on NICE criteria [5], the diagnoses of OSC or PCS were denoted by the prevalence of at least one persistent symptom or sign. Overall, the presence of one or more symptoms in patients was recorded from 20 studies during long COVID [10,12-15,18,23,26,30,33$35,37,39,41-43,45-47]$, with two studies presenting longitudinal data [13,45]. OSC was recorded in 9 distinct studies, with a mean prevalence of $59 \%$ and a range from $14 \%$ to $87 \%$. As for PCS, a prevalence of $62 \%$ for at least one symptom was identified from a total of 11 studies, with a range between $18 \%$ and $89 \%$. Figure 2 depicts the reported prevalences of these two long COVID phases. 


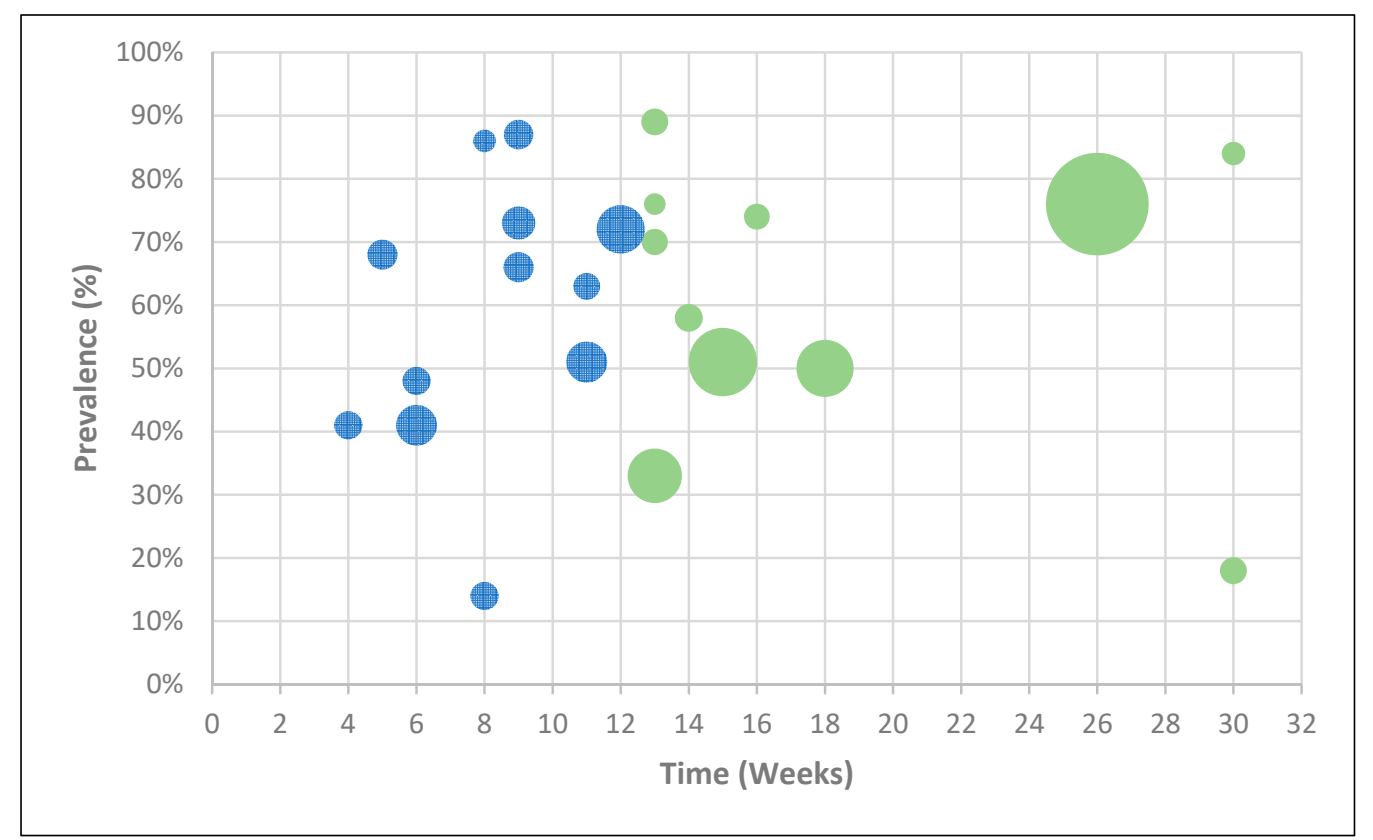

Figure 2. Bubble chart of the reported prevalences of the two long COVID phases (ongoing symptomatic COVID-19 in blue; post-COVID-19 syndrome in green), where the size of each bubble is proportional to the study sample size.

\subsection{Symptomatology}

Figure 3 provides an overview of the mean prevalence proportions of OCS and PSC symptoms across body symptoms, and Table 4 details the prevalence ranges and number of assessment timepoints involved.

\subsubsection{Ongoing Symptomatic COVID-19}

The most prevalent symptom in patients with OSC was fatigue with a mean prevalence of $43 \%$ (range: $5-83 \%$ ). Sleep disorders were also highly prevalent at 36\% (10-69\%), with dyspnoea $(31 \% ; 2-64 \%)$ and cough $(26 \% ; 5-45 \%)$ reported as the most common respiratory symptoms. Other symptoms identified in patients between 4-12 weeks included arthralgia $(23 \% ; 10-48 \%)$, myalgia $(18 \%$; $1-32 \%)$, chest pain $(17 \% ; 3-35 \%)$, headache $(17 \% ; 4-36 \%)$, fever $(15 \% ; 1-51 \%)$, expectoration $(14 \%$; $1-25 \%)$, weight loss $(13 \% ; 6-17 \%)$, skin problems $(12 \% ; 8-15 \%)$, anosmia $(11 \% ; 2-21 \%)$, ageusia $(11 \% ; 1-25 \%)$, and confusion $(11 \% ; 9-14 \%)$. Less common manifestations were eye irritation $(8 \% ; 4-11 \%)$, diarrhoea $(8 \% ; 1-18 \%)$, throat pain $(6 \% ; 1-17 \%)$, palpitations $(6 \% ; 2-11 \%)$, inappetence $(5 \% ; 1-9 \%)$, chest tightness $(4 \%$; $1-6 \%)$, nausea $(2 \% ; 1-6 \%)$, and peptic ulcer $(2 \% ; 1-3 \%)$.

\subsubsection{Post-COVID-19 Syndrome}

Fatigue also presented as the most common symptom in PCS patients at $44 \%(10-71 \%)$, with dyspnoea, myalgia, and sleep disorder prevalent at a mean of $40 \%(6-73 \%), 34 \%$ $(2-86 \%)$, and $33 \%(18-57 \%)$, respectively. Other symptoms reported in patients over 12 weeks post-disease onset included cough (22\%; 3-59\%), hair loss $(20 \%$; 6-29\%), palpitations $(20 \% ; 4-62 \%)$, arthralgia $(13 \% ; 6-29 \%)$, throat pain $(12 \% ; 3-29 \%)$, anosmia $(10 \%$; $5-13 \%)$, and chest pain $(10 \% ; 1-22 \%)$. Fever $(8 \% ; 1-20 \%)$, ageusia $(8 \% ; 2-15 \%)$, and skin problems $(6 \% ; 3-12 \%)$ were less commonly reported. 


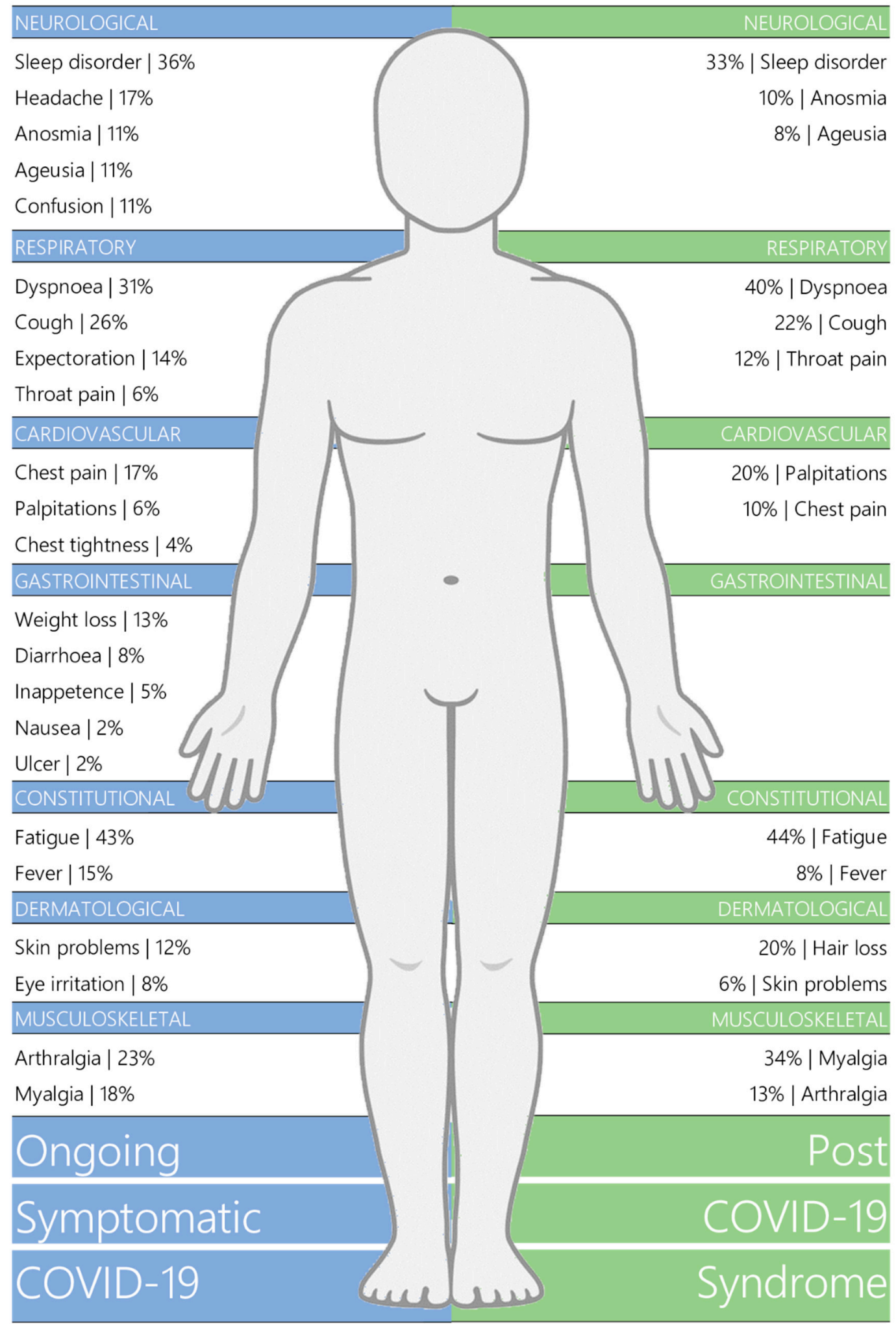

Figure 3. Body Chart of Long COVID Symptomatology. 
Table 4. Symptom Prevalence of Long COVID Patients.

\begin{tabular}{|c|c|c|c|c|c|c|c|c|c|c|}
\hline & \multicolumn{5}{|c|}{ Ongoing Symptomatic COVID-19 } & \multicolumn{5}{|c|}{ Post-COVID-19 Syndrome } \\
\hline & $\bar{X}$ & SD & $\mathbf{N}$ & Min. & Max. & $\bar{X}$ & SD & $\mathbf{N}$ & Min. & Max. \\
\hline \multicolumn{11}{|l|}{ Constitutional } \\
\hline Fatigue & $43 \%$ & 24 & 19 & $5 \%$ & $83 \%$ & $44 \%$ & 19 & 16 & $10 \%$ & $71 \%$ \\
\hline Fever & $14 \%$ & 18 & 8 & $1 \%$ & $51 \%$ & $8 \%$ & 8 & 7 & $1 \%$ & $20 \%$ \\
\hline \multicolumn{11}{|l|}{ Respiratory } \\
\hline Dyspnoea & $31 \%$ & 19 & 25 & $2 \%$ & $64 \%$ & $40 \%$ & 21 & 15 & $6 \%$ & $73 \%$ \\
\hline Cough & $26 \%$ & 13 & 19 & $5 \%$ & $45 \%$ & $22 \%$ & 16 & 16 & $3 \%$ & $59 \%$ \\
\hline Expectoration & $13 \%$ & 8 & 7 & $1 \%$ & $25 \%$ & - & - & - & - & - \\
\hline Throat pain & $6 \%$ & 6 & 7 & $1 \%$ & $17 \%$ & $12 \%$ & 9 & 6 & $3 \%$ & $29 \%$ \\
\hline \multicolumn{11}{|l|}{ Neurological } \\
\hline Sleep disorder & $36 \%$ & 25 & 5 & $10 \%$ & $69 \%$ & $33 \%$ & 13 & 11 & $18 \%$ & $57 \%$ \\
\hline Headache & $17 \%$ & 8 & 10 & $4 \%$ & $36 \%$ & - & - & - & - & - \\
\hline Anosmia & $11 \%$ & 7 & 9 & $2 \%$ & $21 \%$ & $10 \%$ & 3 & 8 & $5 \%$ & $13 \%$ \\
\hline Ageusia & $11 \%$ & 9 & 8 & $1 \%$ & $25 \%$ & $8 \%$ & 4 & 7 & $2 \%$ & $15 \%$ \\
\hline Confusion & $11 \%$ & 3 & 3 & $9 \%$ & $14 \%$ & - & - & - & - & - \\
\hline \multicolumn{11}{|l|}{ Cardiovascular } \\
\hline Chest pain & $17 \%$ & 11 & 9 & $3 \%$ & $35 \%$ & $10 \%$ & 6 & 11 & $1 \%$ & $22 \%$ \\
\hline Palpitations & $6 \%$ & 4 & 5 & $2 \%$ & $11 \%$ & $20 \%$ & 28 & 4 & $4 \%$ & $62 \%$ \\
\hline Chest tightness & $4 \%$ & 3 & 3 & $1 \%$ & $6 \%$ & - & - & - & - & - \\
\hline \multicolumn{11}{|l|}{ Gastrointestinal } \\
\hline Weight loss & $13 \%$ & 6 & 3 & $6 \%$ & $17 \%$ & - & - & - & - & - \\
\hline Diarrhoea & $8 \%$ & 5 & 10 & $1 \%$ & $18 \%$ & - & - & - & - & - \\
\hline Inappetence & $5 \%$ & 4 & 4 & $1 \%$ & $9 \%$ & - & - & - & - & - \\
\hline Nausea & $2 \%$ & 2 & 5 & $1 \%$ & $6 \%$ & - & - & - & - & - \\
\hline Ulcer & $2 \%$ & 1 & 3 & $1 \%$ & $3 \%$ & - & - & - & - & - \\
\hline \multicolumn{11}{|l|}{ Musculoskeletal } \\
\hline Arthralgia & $23 \%$ & 13 & 7 & $10 \%$ & $48 \%$ & $13 \%$ & 11 & 4 & $6 \%$ & $29 \%$ \\
\hline Myalgia & $18 \%$ & 10 & 9 & $1 \%$ & $32 \%$ & $34 \%$ & 31 & 9 & $2 \%$ & $86 \%$ \\
\hline \multicolumn{11}{|l|}{ Dermatological } \\
\hline Skin problems & $12 \%$ & 4 & 3 & $8 \%$ & $15 \%$ & $6 \%$ & 4 & 4 & $3 \%$ & $12 \%$ \\
\hline Eye irritation & $8 \%$ & 3 & 4 & $4 \%$ & $11 \%$ & - & - & - & - & - \\
\hline Hair loss & - & - & - & - & - & $20 \%$ & 9 & 5 & $6 \%$ & $29 \%$ \\
\hline
\end{tabular}

$\overline{\mathrm{X}}=$ mean. $\mathrm{SD}=$ standard deviation. $\mathrm{N}=$ number of assessment timepoints. Min. $=$ minimum. Max. $=$ maximum.

\subsection{Respiratory Functioning}

\subsubsection{Pulmonary Functioning}

Table 5 summarises the prevalence of abnormal pulmonary function parameters across included studies, which include forced expiratory volume in the first second $\left(\mathrm{FEV}_{1}\right)$, forced vital capacity (FVC), the $\mathrm{FEV}_{1} / \mathrm{FVC}$ ratio, and diffusion capacity for carbon monoxide $\left(\mathrm{DL}_{\mathrm{CO}}\right)$. During the OSC phase, $\mathrm{FEV}_{1}$ values below the predicted normal were identified in a mean of $15 \%(9-21 \%)$ of patients. Abnormal FVC scores averaged a prevalence of $12 \%$ (7-21\%), and $\mathrm{FEV}_{1} / \mathrm{FVC}$ and $\mathrm{DL}_{\mathrm{CO}}$ impairments were identified in $6 \%(1-11 \%)$ and $44 \%$ (24-53\%) of patients, respectively. During the PSC phase, the mean prevalence of abnormal $\mathrm{FEV}_{1}$ was $11 \%(5-17 \%)$, and those of FVC, FE1/FVC ratio, and DLCO were $11 \%(1-19 \%)$, $7 \%(6-8 \%)$, and $32 \%(20-46 \%)$, respectively.

\subsubsection{Lung Imaging}

Lung imaging was performed at 15 assessment points using computed tomography $(\mathrm{CT} ; n=6)$, high-resolution CT (HRCT; $n=6)$, chest radiography (CXR; $n=5)$, and / or magnetic resonance imaging (MRI; $n=1$ ). Overall, abnormal imaging patterns were observed in $34 \%(2-60 \%)$ of patients with OSC, with specific abnormalities including ground-glass opacity $(28 \% ; 1-59 \%)$ and fibrosis $(19 \% ; 5-44 \%)$ (Table 5). During the PCS phase, a prevalence of $28 \%(13-53 \%)$ was identified for abnormal patterns; ground-glass opacity was the most prevalent abnormality at $24 \%(2-67 \%)$, with reticulation $(11 \% ; 1-24 \%)$, 
fibrosis $(7 \% ; 2-20 \%)$, and consolidation $(3 \% ; 1-7 \%)$ also recorded in a subset of patients (Table 5).

Table 5. Prevalence of Pulmonary and Cognitive Functioning, Psychological Burden, and Quality of Life.

\begin{tabular}{|c|c|c|c|c|c|c|c|c|c|c|}
\hline & \multicolumn{5}{|c|}{ Ongoing Symptomatic COVID-19 } & \multicolumn{5}{|c|}{ Post-COVID-19 Syndrome } \\
\hline & $\overline{\mathbf{X}}$ & SD & $\mathbf{N}$ & Min. & Max. & $\overline{\mathbf{X}}$ & SD & $\mathbf{N}$ & Min. & Max. \\
\hline \multicolumn{11}{|c|}{ Pulmonary Functioning } \\
\hline $\mathrm{FEV}_{1}<80 \%$ predicted & $15 \%$ & 5 & 5 & $9 \%$ & $21 \%$ & $11 \%$ & 6 & 4 & $5 \%$ & $17 \%$ \\
\hline FVC $<80 \%$ predicted & $12 \%$ & 5 & 5 & $7 \%$ & $21 \%$ & $11 \%$ & 9 & 4 & $1 \%$ & $19 \%$ \\
\hline $\mathrm{FEV}_{1} / \mathrm{FVC}<0.7$ & $6 \%$ & 4 & 4 & $1 \%$ & $11 \%$ & $7 \%$ & 1 & 3 & $6 \%$ & $8 \%$ \\
\hline $\begin{array}{c}\mathrm{DL}_{\mathrm{CO}}<80 \% \text { predicted } \\
\text { Chest Imaging }\end{array}$ & $44 \%$ & 14 & 4 & $24 \%$ & $53 \%$ & $32 \%$ & 11 & 4 & $20 \%$ & $46 \%$ \\
\hline Abnormal pattern(s) & $34 \%$ & 25 & 5 & $2 \%$ & $60 \%$ & $28 \%$ & 17 & 5 & $13 \%$ & $53 \%$ \\
\hline Ground-glass opacity & $28 \%$ & 29 & 3 & $1 \%$ & $59 \%$ & $24 \%$ & 26 & 6 & $2 \%$ & $67 \%$ \\
\hline Fibrosis & $19 \%$ & 22 & 3 & $5 \%$ & $44 \%$ & $7 \%$ & 9 & 4 & $2 \%$ & $20 \%$ \\
\hline Reticulation & - & - & - & - & - & $11 \%$ & 12 & 3 & $1 \%$ & $24 \%$ \\
\hline Consolidation & - & - & - & - & - & $3 \%$ & 3 & 3 & $1 \%$ & $7 \%$ \\
\hline \multicolumn{11}{|l|}{ Cognitive Impairments } \\
\hline Cognitive impairment & $20 \%$ & 11 & 5 & $2 \%$ & $28 \%$ & $15 \%$ & 6 & 5 & $5 \%$ & $22 \%$ \\
\hline $\begin{array}{l}\text { Concentration } \\
\text { issues/Attention issues }\end{array}$ & - & - & - & - & - & $30 \%$ & 9 & 5 & $21 \%$ & $43 \%$ \\
\hline Memory impairment & - & - & - & - & - & $35 \%$ & 16 & 6 & $6 \%$ & $48 \%$ \\
\hline \multicolumn{11}{|l|}{ Psychological Disorder } \\
\hline Anxiety & $28 \%$ & 18 & 4 & $14 \%$ & $53 \%$ & $34 \%$ & 21 & 8 & $6 \%$ & $62 \%$ \\
\hline Depression & $25 \%$ & 15 & 3 & $15 \%$ & $42 \%$ & $32 \%$ & 24 & 9 & $4 \%$ & $76 \%$ \\
\hline Post-traumatic stress & - & - & - & - & - & $18 \%$ & 12 & 3 & $6 \%$ & $31 \%$ \\
\hline \multicolumn{11}{|l|}{ Quality of Life } \\
\hline Decreased quality of life & $40 \%$ & 15 & 3 & $23 \%$ & $53 \%$ & $57 \%$ & 9 & 3 & $51 \%$ & $67 \%$ \\
\hline Decrease in usual activities & - & - & - & - & - & $23 \%$ & 17 & 4 & $2 \%$ & $37 \%$ \\
\hline Mobility issues & $51 \%$ & 15 & 3 & $37 \%$ & $67 \%$ & $32 \%$ & 25 & 3 & $7 \%$ & $56 \%$ \\
\hline Pain or discomfort & - & - & - & - & - & $36 \%$ & 11 & 3 & $27 \%$ & $48 \%$ \\
\hline Depression/Anxiety & - & - & - & - & - & $27 \%$ & 14 & 4 & $14 \%$ & $46 \%$ \\
\hline Issues with self-care & - & - & - & - & - & $10 \%$ & 7 & 4 & $1 \%$ & $17 \%$ \\
\hline
\end{tabular}

$\overline{\mathrm{X}}=$ mean. $\mathrm{SD}=$ standard deviation. $\mathrm{N}=$ number of assessment timepoints. Min. $=$ minimum. Max. $=$ maximum. FEV $1=$ forced expiratory volume in one second. FVC = forced vital capacity. $\mathrm{DL}_{\mathrm{CO}}=$ lung diffusion capacity for carbon monoxide.

\subsection{Cognitive Functioning}

Data on cognitive impairments were available at both phases of long COVID from a total of 10 distinct timepoints [17-19,22,33,36,40]. Data regarding specific modalities of cognition, such as memory, concentration, and attention were available for PCS studies only $[14,21,22,33,40]$ and are presented in Table 5 . A mean proportion of $20 \%(2-28 \%)$ of patients was reported to have cognitive impairment during the OSC phase, and 15\% (5-22\%) during PCS. Both concentration or attention issues and memory deficits were prevalent at $30 \%(21-43 \%)$ and $35 \%(6-48 \%)$, respectively, in patients with PCS.

\subsection{Mental Health \& Quality of Life}

During the OSC phase, anxiety and depression were reported in a mean of $28 \%$ (14-53\%) and $25 \%(15-42 \%)$, respectively (Table 5). 40\% (23-53\%) of patients also expressed a decreased quality of life. The EQ-5D-5L was utilised to assess the quality of life data, with this measure incorporating sub-scales to explore five dimensions of quality of life [50]. Mobility issues were reported in a mean of $51 \%(37-67 \%)$ of patients who completed the EQ-5D-5L assessment during OSC, with insufficient data available for the remaining dimensions.

The PCS phase seemed to have higher mean prevalences of anxiety $(34 \% ; 6-62 \%)$ and depression $(32 \% ; 4-76 \%)$, whilst post-traumatic stress was also prevalent in $18 \%(6-31 \%)$ of patients. A decreased quality of life was recorded in 57\% (51-67\%) of the samples, with the EQ-5D-5L sub-scales identifying the following prevalence proportions: pain or discomfort 
$(36 \% ; 27-48 \%)$, mobility issues $(32 \% ; 7-56 \%)$, depression or anxiety $(27 \% ; 14-46 \%)$, a decrease in usual activities (23\%; $2-37 \%)$, and issues with self-care $(10 \% ; 1-17 \%)$ (Table 5).

\section{Discussion}

\subsection{Statement of Principal Findings}

The aim of this systematic review was to compare the two phases of long COVID, namely OSC (signs and symptoms from 4 to 12 weeks since initial infection) and PCS (signs and symptoms beyond 12 weeks), with respect to symptomatology, abnormal cognitive and respiratory functioning, psychological burden, and quality of life. Overall, findings indicate that the prevalence proportions of OSC and PCS were highly variable across studies, reflecting the non-probabilistic sampling of included studies and differences in hospitalisation status. Reported symptoms covered a wide range of body systems, with a general overlap in frequency ranges between the two long COVID phases. Fatigue and sleep disorders seemed to have comparably high prevalences. Symptoms, such as arthralgia, fever, and chest pain appeared less prevalent in PCS, whilst myalgia, palpitations, and dyspnoea seemed to be more frequently reported during this phase. Data on expectoration, chest tightness, headache, confusion, gastrointestinal issues, and eye irritation was only available for the OSC phase $[13,16,17,22,25,26,28,33,34,45]$, whereas hair loss was only reported in patients with PCS [14,21,23,31,47]. In terms of cognitive impairment, there seemed to be a slightly lower mean prevalence in the PCS phase, with specific data on concentration, attention, and memory being unavailable for the initial long COVID phase. Even though they also had overlapping frequencies, abnormalities in lung function and imaging seemed to have higher frequencies in OSC, whilst anxiety, depression, and poor quality of life seemed more frequent in PCS. Post-traumatic stress was only mentioned in PCS studies $[11,39,43]$.

Overall, findings would suggest that OSC and PCS are a disease continuum with marked clinical overlap as opposed to discrete, easily distinguishable phases. However, results suggest the possibility that OSC may have a higher burden of somatic disease, while PCS may be characterised by a relatively higher psychosocial burden. However, in general, the quality of the evidence was moderate, and many symptoms were only reported in a subset of patients. Therefore, further research is needed to better understand the complex interplay between somatic and psychosocial manifestations in long COVID.

\subsection{Strengths and Weaknesses of the Study}

A strength of the study is the novel approach to the characterisation of long COVID by considering the OSC and PCS phases, which NICE separated as potentially distinct entities [5] but had not yet been systematically characterised. Another robust aspect of this review is the collation of a total of 39 studies conducted in 17 different countries, which captures the global nature of the COVID-19 pandemic.

However, the major limitation of the study resides in the lack of inter-study consistency regarding assessment methods for symptomatology and functional impairments. Many of the studies denoted symptom presence or absence using self-report tools, which are affected by self-report biases [51]. Standardised scales were also utilised, however, there was no consistency in the selected scales with fatigue alone quantified by five distinct objective scales: the Chalder Fatigue Scale [42], the Fatigue Severity Scale [36], the PROMIS [26], and SF-36 [10] scales, and a previously validated unnamed scale [39]. This poor inter-study consistency may compromise the validity of the findings, with scales potentially being more or less sensitive or even assessing distinct sub-domains of a symptom. Abnormal patterns in chest imaging were also highly heterogeneous through the mixed use of chest X-ray, regular $\mathrm{CT}$, high-resolution $\mathrm{CT}$, and magnetic resonance imaging. Due to the limited data available, differences in assessment tools were not addressed in the eligibility screening phase of the review. Overall, the lack of inter-study consistency in methodology may explain the large ranges observed in the data. The moderate quality of the data acquired from the included studies must also be acknowledged in relation to the wide-ranging 
prevalence findings. An average AXIS score of $16.9( \pm 2.0)$ for the studies suggests that the results should be interpreted with caution [9].

\subsection{Strengths and Weaknesses in Relation to Other Studies}

Although the number of reviews attempting to characterise long COVID is exponentially increasing [49,52-56], many of those published present a narrative, rather than systematic, discussion of the findings. In addition to adding value by characterising long COVID separately by OSC and PCS phases, our study offers a structured systematic overview of the long-term effects of COVID-19.

Another point of note regarding the present review is the inclusion of multisystemrelated symptoms and impairments. While previous reviews have focused solely on neurological or respiratory functioning [57-60], our review provides a more comprehensive and collective characterisation of long COVID and further evidences its heterogenous nature. We acknowledge, however, that our review is not fully comprehensive. For example, Nalbandian et al. [52] narratively described haematologic, renal, and endocrine post-acute COVID-19 complications, and these body systems were not incorporated into the present review's literature search. Another potential limitation of the current review was the fact that patient hospitalisation status or acute phase history were not taken into account when characterising the signs and symptoms of OSC and PCS. While primary data for these characteristics were indeed presented by several studies $[21,27,40]$, there were insufficient data available to provide a comprehensive distinction of patients' characteristics with respect to them.

\subsection{Meaning of the Study}

This systematic review provides clinicians, other healthcare professionals, and policymakers with a comprehensive, yet concise, characterisation of the two phases of long COVID, namely OSC and PCS. Overall, the findings provide a systematic description of the typical clinical profile of long COVID patients and could enhance the understanding of the condition, thereby potentially resulting in better treatment options and management of symptoms, and implementation of policies that allow long COVID patients to receive the best possible care. The suggested higher relative importance of psychosocial manifestations in the PCS phase may inform more holistic assessment and treatment strategies, including psychological and psychosocial supports. Additionally, the frequent presence of psychological distress may be linked to several reported symptoms, with a range of psychological disorders often associated with hair loss [61], sleep disorders [62], gastrointestinal issues [63], pain [64], and cardiovascular symptoms [64]. Establishing potential associations will further enhance patient care by enabling to cluster signs and symptoms, and characterise several 'subtypes' of long COVID.

\subsection{Unanswered Questions and Future Research}

Due to the observational nature of the evidence and a very limited longitudinal follow-up in the included studies, we cannot infer how symptoms evolved over time (i.e., whether symptoms increased or decreased with time). While more longitudinal research efforts are emerging at the time of writing [65], further research integrating longitudinal designs is needed to better establish the manifestations in long COVID over time. Further characterisation is needed regarding the potential impact of acute phase presentation, hospitalisation status, medication, vaccination status, age, sex, education, socioeconomic status, occupation, and baseline physical and psychological/psychiatric comorbidities on the risk of developing long COVID. In addition, there is scope for future studies linking long COVID clinical profiles to respective physiological and immunological profiles, to see whether they align in the pathophysiology of long COVID. Finally, improved consistency in symptomatic assessment strategies across future studies may result in a better level of evidence. Addressing all these gaps could ultimately help clinicians enhance symptom management and treatment. 
Supplementary Materials: The following are available online at https: / www.mdpi.com/article/ $10.3390 /$ jcm10245913/s1, Supplementary Data S1. Search Strategy, Supplementary Data S2. Data extraction tool.

Author Contributions: G.J.: Conceptualisation, Methodology, Software, Validation, Formal analysis, Investigation, Data curation, Writing-Original draft, Writing - review and editing, Visualisation. A.M.: Conceptualisation, Methodology, Validation, Investigation, Writing-review and editing, Project administration, Funding acquisition. F.X.: Software, Writing-review and editing, Visualisation. D.M.: Data curation. R.R.-O.: Conceptualisation, Methodology, Validation, Data curation, Writing-review and editing, Supervision, Project administration, Funding acquisition. All authors have read and agreed to the published version of the manuscript.

Funding: Science Foundation Ireland COVID-19 Programme-20/COV/8493.

Institutional Review Board Statement: Not applicable.

Informed Consent Statement: Not applicable.

Conflicts of Interest: The authors declare there is no conflicts of interest.

\section{References}

1. Cucinotta, D.; Vanelli, M. WHO Declares COVID-19 a Pandemic. Acta Biomed. 2020, 91, 157-160. [CrossRef] [PubMed]

2. World Health Organisation. WHO Coronavirus (COVID-19) Dashboard: World Health Organisation. 2021. Available online: https:/ / covid19.who.int/ (accessed on 5 December 2021).

3. Grant, M.C.; Geoghegan, L.; Arbyn, M.; Mohammed, Z.; McGuinness, L.; Clarke, E.L.; Wade, R.G. The Prevalence of Symptoms in 24,410 Adults Infected by the Novel Coronavirus (SARS-CoV-2; COVID-19): A Systematic Review and Meta-Analysis of 148 Studies from 9 Countries. PLoS ONE 2020, 15, e0234765. [CrossRef]

4. Pijls, B.G.; Jolani, S.; Atherley, A.; Derckx, R.T.; Dijkstra, J.I.R.; Franssen, G.H.L.; Hendriks, S.; Richters, A.; Venemans-Jellema, A.; Zalpuri, S.; et al. Demographic Risk Factors for COVID-19 Infection, Severity, ICU Admission and Death: A Meta-Analysis of 59 Studies. BMJ Open 2021, 11, e044640. [CrossRef] [PubMed]

5. National Institute for Health and Care Excellence. COVID-19 Rapid Guideline: Managing COVID-19; NICE: London, UK, 2021.

6. Amin-Chowdhury, Z.; Ladhani, S.N. Causation or Confounding: Why Controls Are Critical for Characterizing Long COVID. Nat. Med. 2021, 27, 1129-1130. [CrossRef]

7. Jennings, G.; Romero-Ortuño, R.; Monaghan, A.; Xue, F. Long COVID: A Systematic Review of Chronic Fatigue and Other Persistent Symptoms Following Acute Phase Recovery: PROSPERO. 2021. Available online: www.crd.york.ac.uk/prospero/ display_record.php?ID=CRD42021247846 (accessed on 1 March 2021).

8. Moher, D.; Liberati, A.; Tetzlaff, J.; Altman, D.G.; The PRISMA Group. Preferred Reporting Items for Systematic Reviews and Meta-Analyses: The PRISMA Statement. PLoS Med. 2009, 6, e1000097. [CrossRef] [PubMed]

9. Downes, M.J.; Brennan, M.; Williams, H.C.; Dean, R. Development of a Critical Appraisal Tool to Assess the Quality of CrossSectional Studies (AXIS). BMJ Open 2016, 6, e011458. [CrossRef] [PubMed]

10. Arnold, D.T.; Hamilton, F.W.; Milne, A.; Morley, A.J.; Viner, J.; Attwood, M.; Noel, A.; Gunning, S.; Hatrick, J.; Hamilton, S.; et al. Patient Outcomes after Hospitalisation with COVID-19 and Implications for Follow-up: Results from a Prospective UK Cohort. Thorax 2020, 76, 399-401. [CrossRef] [PubMed]

11. Bellan, M.; Soddu, D.; Balbo, P.E.; Baricich, A.; Zeppegno, P.; Avanzi, G.C.; Baldon, G.; Bartolomei, G.; Battaglia, M.; Battistini, S.; et al. Respiratory and Psychophysical Sequelae among Patients with COVID-19 Four Months after Hospital Discharge. JAMA Netw. Open 2021, 4, e2036142. [CrossRef]

12. Carfi, A.; Bernabei, R.; Landi, F.; Gemelli Against COVID-19 Post-Acute Care Study Group. Persistent Symptoms in Patients after Acute COVID-19. JAMA 2020, 324, 603-605. [CrossRef]

13. Carvalho-Schneider, C.; Laurent, E.; Lemaignen, A.; Beaufils, E.; Bourbao-Tournois, C.; Laribi, S.; Flament, T.; Ferreira-Maldent, N.; Bruyère, F.; Stefic, K.; et al. Follow-up of Adults with Noncritical COVID-19 Two Months after Symptom Onset. Clin. Microbiol. Infect. 2020, 27, 258-263. [CrossRef]

14. Cheng, D.; Calderwood, C.; Skyllberg, E.; Ainley, A. Clinical Characteristics and Outcomes of Adult Patients Admitted with COVID-19 in East London: A Retrospective Cohort Analysis. BMJ Open Respir. Res. 2021, 8, e000813. [CrossRef] [PubMed]

15. Chopra, V.; Flanders, S.A.; O'Malley, M.; Malani, A.N.; Prescott, H.C. Sixty-Day Outcomes among Patients Hospitalized with COVID-19. Ann. Intern. Med. 2021, 174, 576-578. [CrossRef]

16. Cortés-Telles, A.; López-Romero, S.; Figueroa-Hurtado, E.; Pou-Aguilar, Y.N.; Wong, A.W.; Milne, K.M.; Ryerson, C.J.; Guenette, J.A. Pulmonary Function and Functional Capacity in COVID-19 Survivors with Persistent Dyspnoea. Respir. Physiol. Neurobiol. 2021, 288, 103644. [CrossRef] [PubMed]

17. Daher, A.; Balfanz, P.; Cornelissen, C.; Müller, A.; Bergs, I.; Marx, N.; Müller-Wieland, D.; Hartmann, B.; Dreher, M.; Müller, T. Follow up of Patients with Severe Coronavirus Disease 2019 (COVID-19): Pulmonary and Extrapulmonary Disease Sequelae. Respir. Med. 2020, 174, 106197. [CrossRef] 
18. D'Cruz, R.F.; Waller, M.D.; Perrin, F.; Periselneris, J.; Norton, S.; Smith, L.-J.; Patrick, T.; Walder, D.; Heitmann, A.; Lee, K.; et al. Chest Radiography Is a Poor Predictor of Respiratory Symptoms and Functional Impairment in Survivors of Severe COVID-19 Pneumonia. ERJ Open Res. 2020, 7, 00655-2020. [CrossRef]

19. De Lorenzo, R.; Conte, C.; Lanzani, C.; Benedetti, F.; Roveri, L.; Mazza, M.G.; Brioni, E.; Giacalone, G.; Canti, V.; Sofia, V.; et al. Residual Clinical Damage after COVID-19: A Retrospective and Prospective Observational Cohort Study. PLoS ONE 2020, 15, e0239570. [CrossRef] [PubMed]

20. Froidure, A.; Mahsouli, A.; Liistro, G.; De Greef, J.; Belkhir, L.; Gérard, L.; Bertrand, A.; Koenig, S.; Pothen, L.; Yildiz, H.; et al. Integrative Respiratory Follow-up of Severe COVID-19 Reveals Common Functional and Lung Imaging Sequelae. Respir. Med. 2021, 181, 106383. [CrossRef] [PubMed]

21. Garrigues, E.; Janvier, P.; Kherabi, Y.; Le Bot, A.; Hamon, A.; Gouze, H.; Doucet, L.; Berkani, S.; Oliosi, E.; Mallart, E.; et al. Post-Discharge Persistent Symptoms and Health-Related Quality of Life after Hospitalization for COVID-19. J. Infect. 2020, 81, e4-e6. [CrossRef] [PubMed]

22. Halpin, S.J.; McIvor, C.; Whyatt, G.; Adams, A.; Harvey, O.; McLean, L.; Walshaw, C.; Kemp, S.; Corrado, J.; Singh, R.; et al. Postdischarge Symptoms and Rehabilitation Needs in Survivors of COVID-19 Infection: A Cross-Sectional Evaluation. J. Med. Virol. 2020, 93, 1013-1022. [CrossRef]

23. Huang, C.; Huang, L.; Wang, Y.; Li, X.; Ren, L.; Gu, X.; Kang, L.; Guo, L.; Liu, M.; Zhou, X.; et al. 6-Month Consequences of COVID-19 in Patients Discharged from Hospital: A Cohort Study. Lancet 2021, 397, 220-232. [CrossRef]

24. Huang, Y.; Tan, C.; Wu, J.; Chen, M.; Wang, Z.; Luo, L.; Zhou, X.; Liu, X.; Huang, X.; Yuan, S.; et al. Impact of Coronavirus Disease 2019 on Pulmonary Function in Early Convalescence Phase. Respir. Res. 2020, 21, 163. [CrossRef] [PubMed]

25. Iqbal, A.; Iqbal, K.; Ali, S.A.; Azim, D.; Farid, E.; Baig, M.D.; Bin Arif, T.; Raza, M. The COVID-19 Sequelae: A Cross-Sectional Evaluation of Post-recovery Symptoms and the Need for Rehabilitation of COVID-19 Survivors. Cureus 2021, 13 , e13080. [CrossRef] [PubMed]

26. Jacobs, L.G.; Paleoudis, E.G.; Bari, D.L.-D.; Nyirenda, T.; Friedman, T.; Gupta, A.; Rasouli, L.; Zetkulic, M.; Balani, B.; Ogedegbe, C.; et al. Persistence of Symptoms and Quality of Life at 35 Days after Hospitalization for COVID-19 Infection. PLoS ONE 2020, 15, e0243882. [CrossRef]

27. Lerum, T.V.; Aaløkken, T.M.; Brønstad, E.; Aarli, B.; Ikdahl, E.; Lund, K.M.A.; Durheim, M.T.; Rodriguez, J.R.; Meltzer, C.; Tonby, K.; et al. Dyspnoea, Lung Function and CT Findings Three Months after Hospital Admission for COVID-19. Eur. Respir. J. 2020, 57, 2003448. [CrossRef]

28. Liang, L.; Yang, B.; Jiang, N.; Fu, W.; He, X.; Zhou, Y.; Ma, W.-L.; Wang, X. Three-Month Follow-Up Study of Survivors of Coronavirus Disease 2019 after Discharge. J. Korean Med. Sci. 2020, 35, e418. [CrossRef] [PubMed]

29. Loerinc, L.B.; Scheel, A.M.; Evans, S.T.; Shabto, J.M.; O'Keefe, G.A.; O'Keefe, J.B. Discharge Characteristics and Care Transitions of Hospitalized Patients with COVID-19. Healthcare 2021, 9, 100512. [CrossRef] [PubMed]

30. Mandal, S.; Barnett, J.; Brill, S.E.; Brown, J.S.; Denneny, E.K.; Hare, S.S.; Heightman, M.; Hillman, T.E.; Jacob, J.; Jarvis, H.C.; et al. 'Long-COVID': A Cross-Sectional Study of Persisting Symptoms, Biomarker and Imaging Abnormalities Following Hosptalisation for COVID-19. Thorax 2020, 76, 396-398. [CrossRef]

31. Miyazato, Y.; Morioka, S.; Tsuzuki, S.; Akashi, M.; Osanai, Y.; Tanaka, K.; Terada, M.; Suzuki, M.; Kutsuna, S.; Saito, S.; et al. Prolonged and Late-Onset Symptoms of Coronavirus Disease 2019. Open Forum Infect. Dis. 2020, 7, ofaa507. [CrossRef]

32. Mo, X.; Jian, W.; Su, Z.; Chen, M.; Peng, H.; Peng, P.; Lei, C.; Chen, R.; Zhong, N.; Li, S. Abnormal Pulmonary Function in COVID-19 Patients at Time of Hospital Discharge. Eur. Respir. J. 2020, 55, 2001217. [CrossRef]

33. Moreno-Pérez, O.; Merino, E.; Leon-Ramirez, J.-M.; Andres, M.; Ramos, J.M.; Arenas-Jiménez, J.; Asensio, S.; Sanchez, R.; Ruiz-Torregrosa, P.; Galan, I.; et al. Post-Acute COVID-19 Syndrome. Incidence and Risk Factors: A Mediterranean Cohort Study. J. Infect. 2021, 82, 378-383. [CrossRef]

34. Osikomaiya, B.; Erinoso, O.; Wright, K.O.; Odusola, A.O.; Thomas, B.; Adeyemi, O.; Bowale, A.; Adejumo, O.; Falana, A.; Abdus-salam, I.; et al. 'Long COVID': Persistent COVID-19 Symptoms in Survivors Managed in Lagos State, Nigeria. BMC Infect. Dis. 2021, 21, 304. [CrossRef]

35. Prieto, M.A.; Prieto, O.; Castro, H.M. Long COVID: Cross-Sectional Study. Rev. Fac. Cien. Med. Univ. Nac. Cordoba. 2021, 78, 33-36.

36. Raman, B.; Cassar, M.P.; Tunnicliffe, E.M.; Filippini, N.; Griffanti, L.; Alfaro-Almagro, F.; Okell, T.; Sheerin, F.; Xie, C.; Mahmod, M.; et al. Medium-Term Effects of SARS-CoV-2 Infection on Multiple Vital Organs, Exercise Capacity, Cognition, Quality of Life and Mental Health, Post-Hospital Discharge. EClinicalMedicine 2021, 31, e100683. [CrossRef]

37. Rosales-Castillo, A.; García de Los Ríos, C.; Mediavilla García, J.D. Persistent Symptoms after Acute COVID-19 Infection: Importance of Follow-up. Med. Clin. 2021, 156, 35-36. [CrossRef]

38. Shah, A.S.; Wong, A.W.; Hague, C.J.; Murphy, D.T.; Johnston, J.C.; Ryerson, C.J.; Carlsten, C. A Prospective Study of 12-Week Respiratory Outcomes in COVID-19-Related Hospitalisations. Thorax 2020, 76, 402-404. [CrossRef]

39. Simani, L.; Ramezani, M.; Darazam, I.A.; Sagharichi, M.; Aalipour, M.A.; Ghorbani, F.; Pakdaman, H. Prevalence and Correlates of Chronic Fatigue Syndrome and Post-Traumatic Stress Disorder after the Outbreak of the COVID-19. J. Neurovirol. 2021, 27, 154-159. [CrossRef] [PubMed]

40. Sykes, D.L.; Holdsworth, L.; Jawad, N.; Gunasekera, P.; Morice, A.H.; Crooks, M.G. Post-COVID-19 Symptom Burden: What Is Long-COVID and How Should We Manage It? Lung 2021, 199, 113-119. [CrossRef] 
41. Taboada, M.; Moreno, E.; Cariñena, A.; Rey, T.; Pita-Romero, R.; Leal, S.; Sanduende, Y.; Rodríguez, A.; Nieto, C.; Vilas, E.; et al. Quality of Life, Functional Status, and Persistent Symptoms after Intensive Care of COVID-19 Patients. Br. J. Anaesth. 2020, 126, e110-e113. [CrossRef] [PubMed]

42. Townsend, L.; Dyer, A.H.; Jones, K.; Dunne, J.; Mooney, A.; Gaffney, F.; O'Connor, L.; Leavy, D.; O’Brien, K.; Dowds, J.; et al. Persistent Fatigue Following SARS-CoV-2 Infection Is Common and Independent of Severity of Initial Infection. PLoS ONE 2020, 15, e0240784. [CrossRef]

43. Venturelli, S.; Benatti, S.V.; Casati, M.; Binda, F.; Zuglian, G.; Imeri, G.; Conti, C.; Biffi, A.M.; Spada, M.S.; Bondi, E.; et al. Surviving COVID-19 in Bergamo Province: A Post-Acute Outpatient Re-Evaluation. Epidemiol. Infect. 2021, 149, E32. [CrossRef] [PubMed]

44. Walle-Hansen, M.M.; Ranhoff, A.H.; Mellingsæter, M.; Wang-Hansen, M.S.; Myrstad, M. Health-Related Quality of Life, Functional Decline, and Long-Term Mortality in Older Patients Following Hospitalisation Due to COVID-19. BMC Geriatr. 2021, 21, 199. [CrossRef]

45. Wang, X.; Xu, H.; Jiang, H.; Wang, L.; Lu, C.; Wei, X.; Liu, J.; Xu, S. Clinical Features and Outcomes of Discharged Coronavirus Disease 2019 Patients: A Prospective Cohort Study. QJM 2020, 113, 657-665. [CrossRef]

46. Wong, A.W.; Shah, A.S.; Johnston, J.C.; Carlsten, C.; Ryerson, C.J. Patient-Reported Outcome Measures after COVID-19: A Prospective Cohort Study. Eur. Respir. J. 2020, 56, 2003276. [CrossRef]

47. Xiong, Q.; Xu, M.; Li, J.; Liu, Y.; Zhang, J.; Xu, Y.; Dong, W. Clinical Sequelae of COVID-19 Survivors in Wuhan, China: A Single-Centre Longitudinal Study. Clin. Microbiol. Infect. 2020, 27, 89-95. [CrossRef] [PubMed]

48. Yu, M.; Liu, Y.; Xu, D.; Zhang, R.; Lan, L.; Xu, H. Prediction of the Development of Pulmonary Fibrosis Using Serial Thin-Section CT and Clinical Features in Patients Discharged after Treatment for COVID-19 Pneumonia. Korean J. Radiol. 2020, $21,746-755$. [CrossRef]

49. Halpin, S.; O'Connor, R.; Sivan, M. Long COVID and Chronic COVID Syndromes. J. Med. Virol. 2020, 93, 1242-1243. [CrossRef] [PubMed]

50. Herdman, M.; Gudex, C.; Lloyd, A.; Janssen, M.; Kind, P.; Parkin, D.; Bonsel, G.; Badia, X. Development and Preliminary Testing of the New Five-Level Version of EQ-5D (EQ-5D-5L). Qual. Life Res. 2011, 20, 1727-1736. [CrossRef] [PubMed]

51. Sleep, C.E.; Petty, J.A.; Wygant, D.B. Framing the Results: Assessment of Response Bias through Select Self-Report Measures in Psychological Injury Evaluations. Psychol. Inj. Law 2015, 8, 27-39. [CrossRef]

52. Nalbandian, A.; Sehgal, K.; Gupta, A.; Madhavan, M.V.; McGroder, C.; Stevens, J.S.; Cook, J.R.; Nordvig, A.S.; Shalev, D.; Sehrawat, T.S.; et al. Post-Acute COVID-19 Syndrome. Nat. Med. 2021, 27, 601-615. [CrossRef]

53. Mendelson, M.; Nel, J.; Blumberg, L.; Madhi, S.A.; Dryden, M.; Stevens, W.; Venter, F.W.D. Long-COVID: An Evolving Problem with an Extensive Impact. S. Afr. Med. J. 2020, 111, 10-12. [CrossRef]

54. Rudroff, T.; Fietsam, A.C.; Deters, J.R.; Bryant, A.D.; Kamholz, J. Post-COVID-19 Fatigue: Potential Contributing Factors. Brain Sci. 2020, 10, 1012. [CrossRef]

55. Mahase, E. Long Covid Could Be Four Different Syndromes, Review Suggests. BMJ 2020, 371, m3981. [CrossRef]

56. Oronsky, B.; Larson, C.; Hammond, T.C.; Oronsky, A.; Kesari, S.; Lybeck, M.; Reid, T.R. A Review of Persistent Post-COVID Syndrome (PPCS). Clin. Rev. Allergy Immunol. 2021, 20, 1-9. [CrossRef]

57. Cares-Marambio, K.; Montenegro-Jiménez, Y.; Torres-Castro, R.; Vera-Uribe, R.; Torralba, Y.; Alsina-Restoy, X.; VasconcelloCastillo, L.; Vilaró, J. Prevalence of Potential Respiratory Symptoms in Survivors of Hospital Admission after Coronavirus Disease 2019 (COVID-19): A Systematic Review and Meta-Analysis. Chronic Respir. Dis. 2021, 18. [CrossRef]

58. Tancheva, L.; Petralia, M.C.; Miteva, S.; Dragomanova, S.; Solak, A.; Kalfin, R.; Lazarova, M.; Yarkov, D.; Ciurleo, R.; Cavalli, E.; et al. Emerging Neurological and Psychobiological Aspects of COVID-19 Infection. Brain Sci. 2020, 10, 852. [CrossRef]

59. Rahman, A.; Niloofa, R.; De Zoysa, I.M.; Cooray, A.D.; Kariyawasam, J.; Seneviratne, S.L. Neurological Manifestations in COVID-19: A Narrative Review. SAGE Open Med. 2020, 8. [CrossRef] [PubMed]

60. Jarrahi, A.; Ahluwalia, M.; Khodadadi, H.; da Silva Lopes Salles, E.; Kolhe, R.; Hess, D.C.; Vale, F.; Kumar, M.; Baban, B.; Vaibhav, K.; et al. Neurological Consequences of COVID-19: What Have We Learned and Where Do We Go from Here? J. Neuroinflammation 2020, 17, 286. [CrossRef]

61. Hadshiew, I.M.; Foitzik, K.; Arck, P.C.; Paus, R. Burden of Hair Loss: Stress and the Underestimated Psychosocial Impact of Telogen Effluvium and Androgenetic Alopecia. J. Investig. Dermatol. 2004, 123, 455-457. [CrossRef] [PubMed]

62. van Mill, J.G.; Hoogendijk, W.J.; Vogelzangs, N.; van Dyck, R.; Penninx, B.W. Insomnia and Sleep Duration in a Large Cohort of Patients with Major Depressive Disorder and Anxiety Disorders. J. Clin. Psychiatry 2010, 71, 239-246. [CrossRef] [PubMed]

63. Aguado, A.; García Del Álamo, M. Gastrointestinal Comorbidity and Symptoms Associated with Depression in Patients Aged over 60 Years. Semergen 2020, 46, 27-32. [CrossRef]

64. Jowett, S.; Shevlin, M.; Hyland, P.; Karatzias, T. Posttraumatic Stress Disorder and Persistent Somatic Symptoms during the COVID-19 Pandemic: The Role of Sense of Threat. Psychosom. Med. 2020, 83, 338-344. [CrossRef] [PubMed]

65. Nehme, M.; Braillard, O.; Chappuis, F.; Courvoisier, D.S.; Guessous, I. Prevalence of Symptoms More Than Seven Months after Diagnosis of Symptomatic COVID-19 in an Outpatient Setting. Ann. Intern. Med. 2021, 174, 1252-1260. [CrossRef] [PubMed] 